\title{
Sequence Alignment Algorithms for Intrusion Detection in the Internet of Things
}

\author{
M. Kalinin and V. Krundyshev* \\ Peter the Great St. Petersburg Polytechnic University, \\ 29 Polytechnicheskaya Str, 195251 St.Petersburg, RUSSIA
}

(Received 18 August, 2020)

\begin{abstract}
The paper reviews the intrusion detection approach based on bioinformatics algorithms for alignment and comparing of the nucleotide sequences. Sequence alignment is a natureclose computational procedure for matching the coded strings by searching for the regions of individual characteristics that are located in the same order. A calculated rank of similarity is used instead of equity checking to estimate the distance between a sequence of the monitored operational acts and a generalized intrusion pattern. Multiple alignment schema is more effective and accurate than the Smith-Waterman local alignment due to ability to find few blocks of similarity. In comparison with a traditional signature-based IDS, it is found that the nature-inspired approach provides the better work characteristics. The experimental study have shown that new approach demonstrates high, 99 percent, level of accuracy.
\end{abstract}

AMS Subject Classification: 94A13, 94A60

Keywords: alignment, bioinformatics, bioinspired, detection, homologue, infrastructure, intrusion, IoT, Mauve, Smith-Waterman, security, sequence, similarity

DOI: https://doi.org/10.33581/1561-4085-2020-23-4-397-404

\section{Introduction}

In recent years, the introduction of the technological concept of the Internet of Things has significantly accelerated the pace of digital transformation of the economy. The use of smart cyberspaces of devices increases the productivity of digital enterprises, the efficiency of business processes and the level of service automation. However, in addition to market advantages, a new technological phenomenon is determining the emergence of new security threats and an increase in the number of the targeted invasions in critical infrastructures.

Intrusion detection systems (IDS) are traditionally applied in information systems and computer networks to automate the monitoring of ongoing security events and their analysis to identify security anomalies and threats [1], [2]. They are used to identify certain types of malicious activity that can disrupt the

\footnotetext{
*E-mail: sci@ibks.spbstu.ru
}

security of the protected infrastructure (for example, network attacks against vulnerable services, attacks aimed at privilege escalation, unauthorized access to critical objects, malicious software).

In modern digital infrastructures deployed on the basis of the Internet of Things, large volumes of transmitted data, new dynamic wireless data transfer protocols, a large amount of system information sent in an open manner, the variability and polymorphism of modern intrusions, and the weak protection of the Internet of Things devices themselves are attracting more and more intruders [3-5]. In such conditions, classical intrusion detection tools, traditionally used to ensure the protection of networks and systems, no longer allow the detection of many urgent threats [6].

We propose a new approach to ensuring the security of smart digital infrastructures built on the concept of the Internet of Things by using a computational approach utilized in bioinformatics. For this purpose, the analysis of bioinformatics-relative sequence alignment 
methods is carried out in Section II; the applicability of the sequence alignment for the analysis of network traffic security and the effectiveness of the bioinformation approach to the intrusion detection is assessed in comparison with the traditional signature-based approach in Section III; and finally, Section IV concludes our research.

\section{Analysis of the bioinformatics algorithms for sequence alignment}

\subsection{The sequence alignment algorithms}

Signature-based intrusion detection methods, in comparison with anomalous-based ones, are characterized by high reliability and relatively low requirements for the computing resources. In this regard, they are simpler and are applied more often when implementing the IDS for light-weight environments [7].

Signature is a set of criteria that are used as a template for detecting signs of attacks in network traffic. Detection using signature matching is to search for a predefined, static sequence of features in a data item (e.g., a network packet). The advantages of this method are the simplicity of defining detection rules and applicability for any network protocols. However, the non-unique pattern can lead to a large number of false positives of this approach. It may also require a large database of templates to identify a polymorphic intrusion, for which the indicative variants of its exploitation are different. The use of more complex, for instance, heuristic, signature-based methods allows the use of logical rules to set threshold values of monitored parameters, the excess of which indicates a violation $[8,9]$. Then, with precise definition of the detection rules, intrusion detection faults are minimized. It becomes possible to identify the polymorphic attacks, but dependence of the detector's accuracy on the complexity, fullness, and correctness of the signature rules database increases. As a result, unknown protocols and new variations of the intrusions and violations may not be detected [10-12].

Bioinformation methods are devoid of these disadvantages. They help the biologists and genetic engineers to determine the similarity (not equity) of the DNA and RNA chains, to detect nucleotide mutations and genetic anomalies. Various genomics methods have been developed that are resistant to the variability of the processed genetic informational structures.

In bioinformatics, nucleotide sequences that are very similar are called homologous. To establish the homology of the sequences being compared, the sequence alignment is applied - the computational procedure for matching sequences by searching for sets and subsets of individual elements or characteristic combinations of elements that are located in the same order [13-15]. A measure of homology is a computed similarity rank which can be used to estimate the distance between a given biocode sequence and a pattern.

There are two kinds of the sequence alignment approaches:

1. Global alignment is an alignment of the entire sequence relative to another. It is used if the sequences are homologous along the entire length [16].

2. Local alignment is a search for homology in parts of sequences. It is used to compare sequences with partial similarity. For local matching, overhanging ends of the sequences are not considered gaps (spaces, or blanks). In addition to mismatches, insertions and deletions within the matched part are also possible [17].

And, as a combination of the above approaches, there is also known the multiple alignment, the alignment of a set of three or more sequences at the same time, in which homologous elements are grouped into blocks [16].

\subsection{The global alignment}

The Needleman-Wunsch global alignment [18], containing possible alignments, is built in 
the form of a matrix in which the elements of one sequence form rows, and the elements of the other sequence form columns. The optimization task of the algorithm is to find the diagonal path between the matrix elements that has the maximum weight. The algorithm recursively constructs the optimal alignment at each stage based on the optimal alignment of the initial fragments of the original sequences obtained at the previous stages.

For two sequences to be aligned $x$ and $y$ with the corresponding elements $x_{i}, 0<i<n$, and $y_{j}, 0<j<m$, a matrix $F$ is constructed. The element $F(i, j)$ of this matrix contains the weight of the best alignment of the initial fragments $x_{1 \ldots i}$ with the length $i$ and $y_{1 \ldots j}$ with the length $j$ of the sequences $x$ and $y$.

The construction begins with assigning a zero value to an element $F(0,0)$ and boundary values $F(i, 0)=-d$ and $F(0, j)=-d$, where $d$ is a predefined penalty. The choice of the value of the penalty is important, as too large a value can lead to the fact that it will be impossible to make an insert, and a small one - to the fact that the alignment will be removed. In addition to statically selected penalties, linear or affine penalties can be used. Then the elements of the matrix are calculated from the first to the last row from left to right, until the entire matrix is filled.

There are three options for calculating the cell $F(i, j)$ :

1. If an element $x_{i}$ of one sequence can be aligned with an element $y_{j}$ of the second sequence, then points $s\left(x_{i}, y_{j}\right)$ for successful alignment from the previously selected substitution matrix $F(i-$ $1, j-1)+s\left(x_{i}, y_{j}\right)$ are added to the weight value $F(i-1, j-1)$.

2. If an element $x_{i}$ of one sequence is matched with a gap "-" in the second sequence, then a penalty $d$ is charged: $F(i-1, j)-d$.

3 . If an element $y_{j}$ of the second sequence is matched with a gap "-" in the first sequence, then a penalty is also charged: $F(i, j-1)-d$.

Optimal alignment is defined as the highest

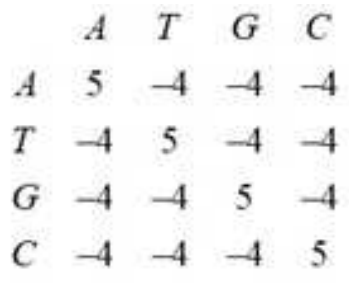

FIG. 1. NUC44 matrix. A, T, G, C are the nucleotide codes.

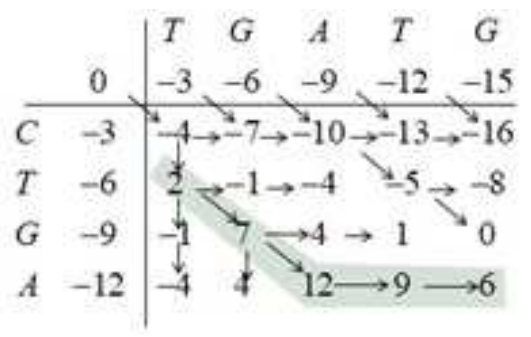

FIG. 2. Example of a global alignment matrix. A sequence with the highest weight is highlighted.

value:

$$
F(i, j)=\max \left\{\begin{array}{c}
F(i-1, j-1)+s\left(x_{i}, y_{j}\right) \\
F(i-1, j)-d \\
F(i, j-1)-d .
\end{array}\right.
$$

The cell value $F(n, m)$ is by definition the best alignment weight of the sequences $x$ and $y$. To build a global alignment, it is necessary to perform a reverse pass - to restore the route from the element $F(n, m)$, following the step marks that were obtained during the construction of the matrix. In this case, the aligned lines are written by adding a pair of characters to the left of the current alignment: $\left|\begin{array}{l}x_{i} \\ y_{j}\end{array}\right|$ if the value is received from the cell $(i-1, j-1) ;\left|\begin{array}{c}x_{i} \\ -\end{array}\right|$ if the value is obtained from a cell $(i-1, j) ;\left|\begin{array}{c}- \\ y_{j}\end{array}\right|$ if the value is from a cell $(i-1, j-1)$. NUC44 is a substitution matrix for this algorithm (Figure 1).

Let us consider two sequences $x=T G A T G, y=C T G A$, and $d=3 . \quad$ The global alignment matrix is shown in Figure 2.

When performing the reverse pass, we obtain 


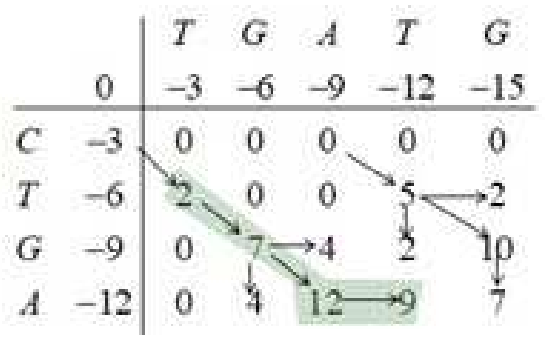

FIG. 3. Example of a local alignment matrix. A sequence with the highest weight is highlighted.

the optimal alignment of the sequences $x$ and $y$ : T G A - -

\subsection{The local alignment}

The Smith-Waterman local alignment [19] is used when it is necessary to find the optimal alignment of subsequences of the original sequences $x$ and $y$. Local alignment is similar to global alignment with two main differences:

1. When the best weight in the matrix $F$ turns out to be negative, zero is written into the cell, which means that there is no path from this cell:

$$
F(i, j)=\max \left\{\begin{array}{c}
0 \\
F(i-1, j-1)+s\left(x_{i}, y_{j}\right) \\
F(i-1, j)-d \\
F(i, j-1)-d .
\end{array}\right.
$$

2. Alignment can end anywhere in the matrix $F$. Then the best alignment weight is not the value $F(n, m)$, but the largest value $F(i, j)$, and the back pass should start from this element.

For an example of the above mentioned sequence, the local alignment matrix is shown in Figure 3.

When performing the reverse pass, we obtain the optimal alignment of the sequences $x$ and $y$ : T G A -

\subsection{The multiple alignment}

The Mauve alignment schema allows for multiple alignments of multiple sequences, reducing the likelihood of misalignments by dividing the sequence into short homologous anchor blocks. A feature of the algorithm is that it is able to find alignments in collinear chains [20].

The Mauve algorithm includes the following steps:

1. Search for local alignments (multiMUMs).

2. Use multi-MUMs to build a backtrack tree.

3. Select a subset of the multi-MUMs to split them into local collinear blocks.

4. Perform additional alignments within the local collinear blocks.

5. Build a backtrack tree for each local collinear block.

Thus, to construct alignments of two sequences, a matrix $F$ is formed, the elements of which are calculated by the formula:

$$
F(i, j)=\max \left\{\begin{array}{c}
0 \\
F(i-1, j-1)+Q\left(x_{i}, y_{j}\right),
\end{array}\right.
$$

where

$Q\left(x_{i}, y_{j}\right)=\left\{\begin{array}{c}0, \text { if } s\left(x_{i}, y_{j}\right) \leq 0 \\ \frac{2 s\left(x_{i}, y_{j}\right)}{s_{a c c}\left(x_{i}\right) \cdot s_{a c c}\left(y_{j}\right)}-s\left(x_{i}, y_{j}\right), \text { elsewhere }\end{array}\right.$ where $s\left(x_{i}, y_{j}\right)$ are the values of the matrices of weights between the characters of the sequences, $s_{a c c}\left(x_{i}\right)$ is the number of occurrences of the character $x_{i}$ in the string $x$.

The evaluation of the alignment result (homology rank) is carried out by the formula:

$M(x, y)=\frac{N_{\text {res }}}{\max (\operatorname{len}(x), \operatorname{len}(y))} \cdot \frac{k}{\left(\frac{N_{x}}{\operatorname{len}(x)}+\frac{N_{y}}{\operatorname{len}(y)}\right)}$,

where $N_{\text {res }}$ is the number of characters in the resulting subsequence, $\operatorname{len}(x)$ and $\operatorname{len}(y)$ are the lengths of the sequences $x$ and $y$, correspondingly, $N_{x}$ and $N_{y}$ are the numbers of characters in the sequences $x$ and $y$ included in the resulting one, $k$ is a coefficient that determines the number of occurrences of the resulting subsequence in 


$$
\begin{array}{ccccc} 
& A & T & G & C \\
A & 91 & -123 & -31 & -114 \\
T & -123 & 91 & -114 & -31 \\
G & -31 & -114 & 100 & -125 \\
C & -114 & -31 & -125 & 100
\end{array}
$$

FIG. 4: HOXD matrix.

\begin{tabular}{c|ccccc}
0 & $T$ & $G$ & $A$ & $T$ & $G$ \\
\hline$C$ & 0 & 0 & 0 & 0 & 0 \\
$T$ & 91 & 0 & 0 & 91 & 0 \\
$G$ & 0 & 473 & 0 & 0 & 473 \\
$A$ & 0 & 0 & 655 & 0 & 0
\end{tabular}

FIG. 5. Example of a multiple alignment matrix. A sequences with the highest weights are highlighted.

the original ones. Thus, when aligning several sequences, the sequence similarity coefficient is calculated and a row proximity matrix is constructed. The substitution matrix for this algorithm is the HOXD matrix (Figure 4). For the previously considered sequences, the alignment matrix is shown in Figure 5. Accordingly, the optimal sequence alignments: T G A and T G.

\section{Comparison of the alignment algorithms}

Overall analysis of the most well-known sequence alignment algorithms applied in bioinformatics (Table 1) allows us to labor out the next findings.

Ensuring accuracy in detecting the intrusions in the IoT is of utmost importance. The highest accuracy, over 90 percent, is provided by the LASTZ, Mauve and Markov model.

An IDS must be able to detect the intrusions quickly. The shortest analysis time for large sequences is demonstrated by the Mauve algorithm.

All the compared alignment algorithms
Table 1. Comparison of sequence alignment algorithms.

\begin{tabular}{ccc}
\hline \hline Algorithm & Alignment type & Accuracy \\
\hline PSI-BLAST [21] & multiple local & 70 \\
FASTA [22] & global & 78 \\
Smith-Waterman [19] & local & 80 \\
Hidden Markov model & multiple local & 90 \\
[23, 24] & & \\
LASTZ [25] & local & 90 \\
Mauve [20] & multiple local & 98 \\
\hline \hline
\end{tabular}

are capable of accepting more than a million pairs as input simultaneously, however, unlike bioinformatics system, for the IDS the size of the input is limited and does not matter much.

Therefore, the Smith-Waterman and Mauve algorithms allow using the smallest sequence windows, they have the higher performance. The Mauve alignment algorithm also provides the highest accuracy of the obtained results.

\section{The experimental results}

Let us consider the results of assessing the effectiveness of the bioinformatics-based approach to detecting the security intrusions with the alignment algorithms using the example of detecting the vulnerability CVE-2018-4878 [26].

This network security threat is a variant of exploiting a use-after-free vulnerability in Adobe Flash Player that allows remote code execution through a corrupted Flash object. The vulnerability is used to download from a compromised web server to a host a malicious shellcode containing the ROKRAT remote administration tool.

An example of a YARN rule for identifying this threat is shown in Figure 6. An example of a network packet dump containing malicious traffic that matches this YARN rule is shown in Figure 7.

As data for the operation of the algorithms, we used network traffic dumps collected at Ubuntu 18.04 environment. Our data set contains half of the anomalous samples, and the training 


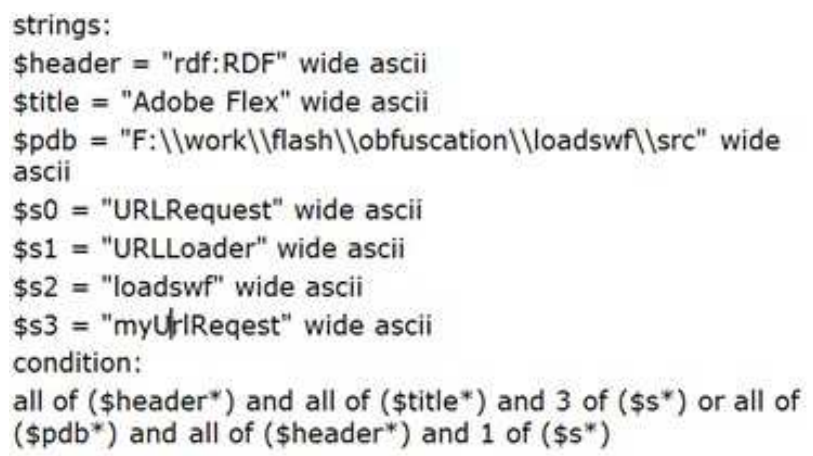

FIG. 6: YARN rule for CVE-2018-4878.

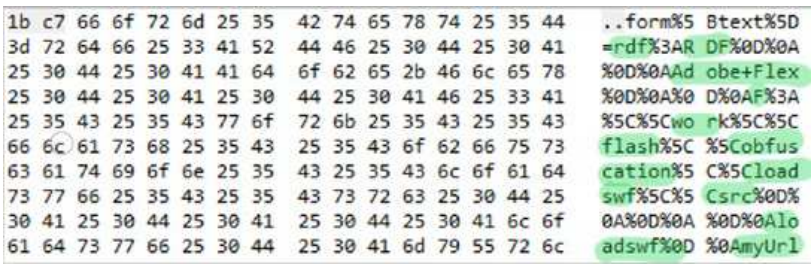

FIG. 7. The network packet dump that matches the YARN rule for CVE-2018-4878.

and test samples are marked in a 70:30 proportion (Table 2).

The method was implemented using the following software: Python, dpkt library, which provides fast analysis of TCP/IP protocol packets, biopython library, which provides capabilities for performing actions with biological sequences, genomes, phylogenetic trees, scikitlearn library, implements machine learning algorithms, which allows us to calculate metrics and visualize the results.

Based on the traffic analysis, abnormal and normal traffic patterns were created. To evaluate the proposed intrusion detection method, it is necessary to determine the boundary of the distance indicator between the matched sequences - $M(x, y)$. To do this, during the

Table 2: Data set characteristics.

\begin{tabular}{ccc}
\hline \hline Type of traffic & Vol. of training set & Vol. of test set \\
\hline Normal & 49,000 & 21,000 \\
Abnormal & 24,500 & 10,500 \\
\hline \hline
\end{tabular}

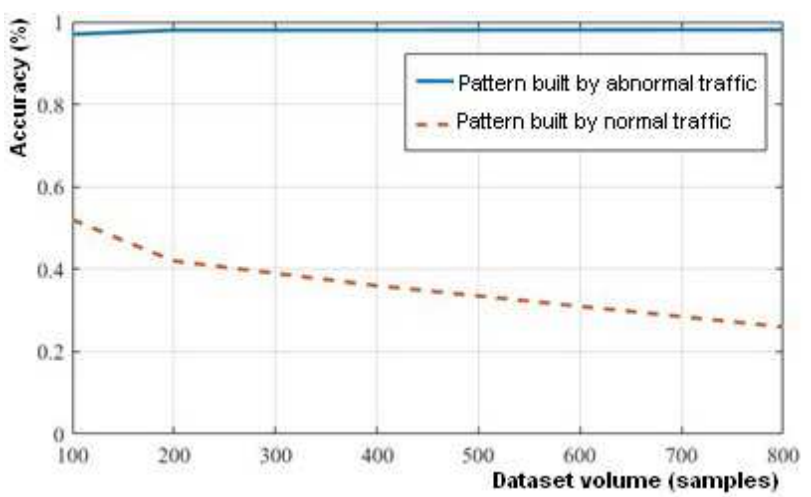

FIG. 8. (color online) The accuracy of the method based on patterns built by normal and abnormal traffic (for sample of CVE-2018-4878).

training stage, the data of the training sample is marked, after which, based on the results, the criteria are formulated by which the detector is triggered (for CVE-2018-4878, the criterion for determining an attack by a normal traffic pattern: $M(x, y) \geq 0.63$, by an abnormal traffic pattern $M(x, y) \geq 0.71)$.

Training stage includes the following steps:

1. For each element of the sample $x$ :

1.1. Conversion to .FA format (this is an accepted in bioinformatics format of codes of nucleotide sequences);

1.2. Carrying out the alignment with the template $y$; $M(x, y)$.

1.3. Getting the rank of alignment

2. Calculation $\min M(x, y)$ among the marked "bad" items.

To select the detection method - according to the pattern of normal or abnormal traffic - the accuracy was measured on a test data set. The Mauve and Smith-Waterman algorithms have been prototyped as the alignment algorithms. Intrusion detection using an anomalous traffic pattern is preferable because of its greater accuracy level (Figure 8).

To analyze the effectiveness of the algorithms, the alignment algorithms and the Suricata IDS [27] were compared. For a comparative assessment, the metrics of accuracy 


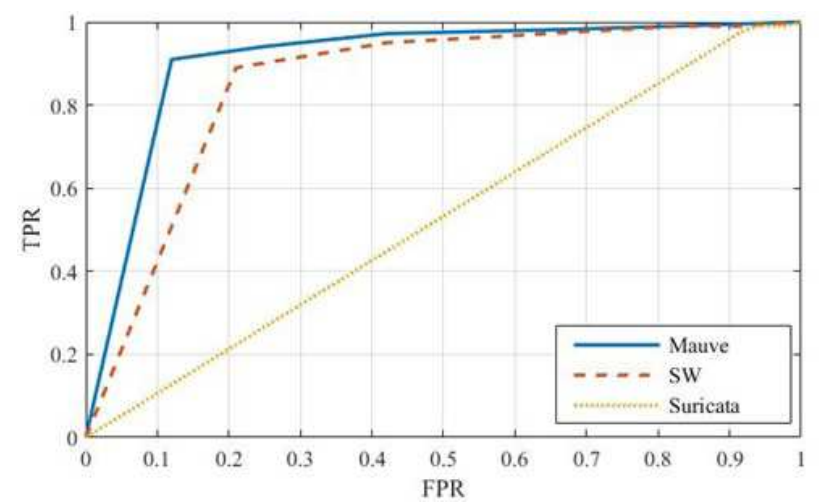

FIG. 9. (color online) ROC curves for assessing the quality of implementations of intrusion detection methods (using the test sample of identifying the CVE-2018-4878 intrusion).

and precision were calculated and compared (Table 3). For comparative visualization of the quality of the studied approaches, the ROC curves were constructed (Figure 9).

Intrusion detection using the Mauve algorithm is more efficient and accurate than the one using the Smith-Waterman algorithm, due to the fact that the Smith-Waterman algorithm is not able to find multiple homologies.

In comparison with the traditional approach (e.g. presented by Suricata IDS), it was found that the bioinformatics-inspired approach provides better operational characteristics than the signature-based IDS.

The obtained results indicate that the developed variants of bioinformatics methods can let us solve the intrusion detection task in the Internet of Things environment protecting it against polymorphic and time-spaced intrusions. Also, the unknown security anomalies can be

Table 3. Comparison of intrusion detection tools (using the example of identifying the CVE-2018-4878 intrusion).

\begin{tabular}{cccc}
\hline \hline Characteristic & Mauve & Smith-Waterman & Suricata \\
\hline Accuracy & 98.4 & 93.6 & 74.9 \\
Precision & 99.0 & 92.7 & 66.6 \\
\hline \hline
\end{tabular}

detected using the similarity of the monitored actions to the generalized patterns of the previously detected incidents.

\section{Conclusion}

Based on the hypothesis that biological sequences are similar to the sequences of transmitted data in the network, a new intrusion detection approach based on bioinformatics algorithms for alignment of the nucleotide sequences has been proposed. The review of the selected alignment algorithms showed the effectiveness of two mechanisms - the Mauve multiple local alignment algorithm and the Smith-Waterman local alignment algorithm.

The created prototypes of testing tools based on bioinformatics algorithms demonstrated high accuracy of the intrusion detection - up to 99 percent. The high quality indicators of sequence alignment algorithms obtained when studying our approach have confirmed that bioinformatics methods can be successfully applied to the intrusion detection tasks in flexible digital infrastructures.

Our further work is targeted at developing the resource optimized version of the suggested method.

\section{Acknowledgement}

The reported study was funded by RFBR according to the research project 18-29-03102. Project results are achieved using the resources of supercomputer center of Peter the Great St.Petersburg Polytechnic University - SCC "Polytechnichesky" (www.spbstu.ru). 


\section{References}

[1] D. H. Lakshminarayana, J. Philips, and N. Tabrizi, in Proceedings - 18th IEEE International Conference on Machine Learning and Applications, ICMLA 2019 (2019), pp. 1122-1129, ISBN 9781728145495.

[2] S. A. V. Jatti and V. J. Kishor Sontif, International Journal of Recent Technology and Engineering 8, 3976 (2019), ISSN 22773878.

[3] A. D. Dakhnovich, D. A. Moskvin, and D. P. Zegzhda, Automatic Control and Computer Sciences 52, 1071 (2018), ISSN 1558108X.

[4] V. V. Platonov and P. O. Semenov, Automatic Control and Computer Sciences 52, 959 (2018), ISSN 1558108X.

[5] F. A. Alaba, M. Othman, I. A. T. Hashem, and F. Alotaibi, Internet of Things security: A survey (2017).

[6] L. Santos, C. Rabadao, and R. Goncalves, in Iberian Conference on Information Systems and Technologies, CISTI (2018), vol. 2018-June, pp. 1-7, ISBN 9789899843486, ISSN 21660735.

[7] J. McHugh, International Journal of Information Security 1, 14 (2001), ISSN 1615-5262.

[8] P. D. Zegzhda, D. S. Lavrova, and A. A. Shtyrkina, Automatic Control and Computer Sciences 52, 936 (2018), ISSN 1558108X.

[9] D. Lavrova, A. Pechenkin, and V. Gluhov, Automatic Control and Computer Sciences 49, 735 (2015), ISSN 1558108X.

[10] E. V. Malyshev, D. A. Moskvin, and D. P. Zegzhda, Automatic Control and Computer Sciences 53, 889 (2019), ISSN 1558108X.

[11] M. Mohammed, A.-S. K. Pathan, M. Mohammed, and A.-S. K. Pathan, in Automatic Defense Against Zero-day Polymorphic Worms in Communication Networks (2013), pp. 47-84.

[12] A. H. Sung and S. Mukkamala, The feature selection and intrusion detection problems (2004).

[13] T. W. Tan and E. Lee, in Beginners Guide to Bioinformatics for High Throughput Sequencing
(2018), pp. 81-115.

[14] H. Li and N. Homer, A survey of sequence alignment algorithms for next-generation sequencing (2010).

[15] Y. A. Markov and M. O. Kalinin, Intellectual intrusion detection with sequences alignment methods, vol. 6258 LNCS (2010), ISBN 3642147054.

[16] Y. Bilu, P. K. Agarwal, and R. Kolodny, in IEEE/ACM Transactions on Computational Biology and Bioinformatics (2006), vol. 3, pp. 408-422, ISSN 15455963.

[17] T. W. Lam, W. K. Sung, S. L. Tam, C. K. Wong, and S. M. Yiu, Bioinformatics 24, 791 (2008), ISSN 13674803.

[18] S. B. Needleman and C. D. Wunsch, Journal of Molecular Biology 48, 443 (1970), ISSN 00222836 .

[19] T. F. Smith and M. S. Waterman, Journal of Molecular Biology 147, 195 (1981), ISSN 00222836 .

[20] A. C. Darling, B. Mau, F. R. Blattner, and N. T. Perna, Genome Research 14, 1394 (2004), ISSN 10889051 .

[21] I. Friedberg, T. Kaplan, and H. Margalit, Protein Science 9, 2278 (2000), ISSN 09618368.

[22] W. R. Pearson, Current Protocols in Bioinformatics 2016, 3.9.1 (2016), ISSN 1934340X.

[23] M. T. Johnson, IEEE Signal Processing Letters 12, 407 (2005), ISSN 10709908.

[24] J. D. Thompson, F. Plewniak, and O. Poch, Nucleic Acids Research 27, 2682 (1999), ISSN 03051048 .

[25] V. P. E. Lenis, M. Swain, and D. M. Larkin, GigaScience 7 (2018), ISSN 2047217X.

[26] CVE-2018-4878 in Common Vulnerabilities and Exposures Description, https://cve.mitre. org/cgi-bin/cvename. cgi ?name $=2018-4878$, accessed: 2020-04-20.

[27] Suricata IDS, suricata-ids.org, accessed: 2020-04-20. 